\title{
Microbiota transplantation
}

Diane E. Hoffmann, Francis B. Palumbo, Jacques Ravel, Virginia Rowthorn, Erik von Rosenvinge

\section{Source}

Diane E. Hoffmann, Francis B. Palumbo, Jacques Ravel, Virginia Rowthorn, Erik von Rosenvinge. (2017). A proposed definition of microbiota transplantation for regulatory purposes. Gut Microbes, vol. 8 (3), 208-213. doi:10.1080/19490976.2017.1293223.

A microbiota transplantation is the transfer of biologic material containing a minimally manipulated ${ }^{1}$ community of microorganisms from a human donor to a human recipient (including autologous use) with the intent of affecting the microbiota of the recipient.

Notes

${ }^{1}$ Minimal manipulation is processing that does not alter the original relevant characteristics of the transferred community of microorganisms. 